\title{
Functional characterization of Plasmodium berghei PSOP25 during ookinete development and as a malaria transmission-blocking vaccine candidate
}

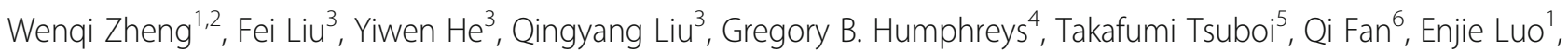
Yaming $\mathrm{CaO}^{3 *}$ and Liwang Cui ${ }^{4}$

\begin{abstract}
Background: Plasmodium ookinete surface proteins as post-fertilization target antigens are potential malaria transmission-blocking vaccine (TBV) candidates. Putative secreted ookinete protein 25 (PSOP25) is a highly conserved ookinete surface protein, and has been shown to be a promising novel TBV target. Here, we further investigated the TBV activities of the full-length recombinant PSOP25 (rPSOP25) protein in Plasmodium berghei, and characterized the potential functions of PSOP25 during the $P$. berghei life-cycle.

Methods: We expressed the full-length P. berghei PSOP25 protein in a prokaryotic expression system, and developed polyclonal mouse antisera and a monoclonal antibody ( $\mathrm{mAb}$ ) against the recombinant protein. Indirect immunofluorescence assay (IFA) and Western blot were used to test the specificity of antibodies. The transmission-blocking (TB) activities of antibodies were evaluated by the in vitro ookinete conversion assay and by direct mosquito feeding assay (DFA). Finally, the function of PSOP25 during Plasmodium development was studied by deleting the psop25 gene.

Results: Both polyclonal mouse antisera and anti-rPSOP25 mAb recognized the PSOP25 proteins in the parasites, and IFA showed the preferential expression of PSOP25 on the surface of zygotes, retorts and mature ookinetes. In vitro, these antibodies significantly inhibited ookinetes formation in an antibody concentration-dependent manner. In DFA, mice immunized with the rPSOP25 and those receiving passive transfer of the anti-rPSOP25 mAb reduced the prevalence of mosquito infection by 31.2 and $26.1 \%$, and oocyst density by 66.3 and $63.3 \%$, respectively. Genetic knockout of the psop25 gene did not have a detectable impact on the asexual growth of $P$. berghei, but significantly affected the maturation of ookinetes and the formation of midgut oocysts.

Conclusions: The full-length rPSOP25 could elicit strong antibody response in mice. Polyclonal and monoclonal antibodies against PSOP25 could effectively block the formation of ookinetes in vitro and transmission of the parasites to mosquitoes. Genetic manipulation study indicated that PSOP25 is required for ookinete maturation in $P$. berghei. These results support further testing of the PSOP25 orthologs in human malaria parasites as promising TBV candidates.
\end{abstract}

Keywords: Plasmodium berghei, PSOP25, Ookinete, Transmission-blocking vaccine

\footnotetext{
* Correspondence: ymcao@mail.cmu.edu.cn

${ }^{3}$ Department of Immunology, College of Basic Medical Sciences, China

Medical University, Shenyang, Liaoning 110001, China

Full list of author information is available at the end of the article
} 


\section{Background}

Malaria remains one of the most prevalent tropical infectious diseases and is endemic in nearly 95 countries and territories around the world, with estimated $>3.2$ billion people being at risk. In 2015, there were approximately 214 million new malaria cases resulting in 438,000 deaths, $~ 80 \%$ of which occurred in Africa [1]. Currently, due to the spread of insecticide-resistant mosquitoes and multidrug-resistant parasites, major malaria control efforts including vector control and chemotherapy are becoming increasingly ineffective [2-4]. These trends highlight the need for developing an integrated malaria control strategy to eliminate malaria transmission. A transmission-blocking vaccine (TBV) targeting the sexual stages of the Plasmodium has the potential to reduce malaria transmission and prevent the spread of resistant parasites. It is predicted that TBV administration can reduce child mortality even in areas of high endemicity [5]. Additionally, TBV can slow down the spread of mutant parasites, which will prolong the effective lives of antimalarial drugs and vaccines [6]. Mathematical models further predict that TBVs will be an effective tool for malaria elimination [7].

TBV is designed to target the Plasmodium antigens expressed during sexual development or Anopheles midgut proteins that interact with sexual stages and allow ookinetes to traverse the Anopheles midgut epithelial cells. Research on TBVs has led to the identification and experimental validation of several potential TBV candidates, but only a few including Pfs48/45 [8, 9], Pfs230 $[10,11]$ and Pfs25 [12] in P. falciparum, and Pvs25 and Pvs28 in $P$. vivax [13], have been found effective in blocking parasite transmission. Investigations on the two 6-cysteine domain protein family members, Pfs $48 / 45$ and Pfs230, have shown that anti-Pfs48/45 monoclonal and polyclonal antibodies in experimental animals can effectively inhibit the transmission of $P$. falciparum to mosquitoes $[9,14,15]$, while Pfs230-raised antibodies are sufficient to block development of the oocysts and competent to induce complement-dependent transmissionblocking (TB) activity [11]. Furthermore, antibodies against both Pfs48/45 and Pfs230 have been detected in natural infections, thereby bringing the potential to boost and/or enhance antibody titers with TBVs against these antigens [16]. Unlike pre-fertilization proteins, post-fertilization antigens are expressed solely after the formation of the zygotes within the mosquito midgut. Concealed from the host's immune system, these antigens have limited diversity among the parasite populations $[17,18]$. The major ookinete surface protein Pfs 25 is a well-characterized $25-\mathrm{kDa}$ glycosyl-phosphatidylinositol (GPI)-anchored protein with four epidermal growth factorlike domains. Pfs25 is involved in adhesion of ookinete and plays an important role in subsequent penetration of the mosquito midgut $[19,20]$. Mouse antiserum against native Pfs25 [21], heterologously expressed Pfs25, or the P. vivax ortholog Pvs25 proteins can effectively inhibit parasite development in mosquitoes [22-24]. Though Pfs25 and Pvs25 provide evidence for the efficacy of post-fertilization antigens in TBVs, more TBV candidate antigens and higher levels of TB activities are needed for an effective deployable vaccine.

With efforts for identifying new TBV candidates, we have recently identified a post-fertilization antigen PSOP25 (PBANKA_111920) in the rodent parasite Plasmodium berghei. Psop25 encodes a 350 amino acid (aa) protein with a signal peptide, and the native protein is predicted to be $40 \mathrm{kDa}$. Psop 25 transcript is highly expressed in ookinetes and occupied in the 99th percentile in the transcriptome of ookinetes [25]. Ookinetespecific expression of this protein was confirmed in our previous study [26]. Antisera from mice immunized with a partial PSOP25 domain (aa 45-245), which included ten predicted antibody epitopes, inhibited ookinete formation by $53.0 \%$ in in vitro ookinete cultures. Mosquitoes fed on this partial PSOP25 domain-immunized mice also resulted in modestly decreased oocyst prevalence $(25.0 \%)$ and significantly reduced oocyst densities (64.3\%) [26], suggesting that PSOP25 could be a new promising target for TBVs. Here we set out to further investigate the TBV activities of the full-length PSOP25 protein in $P$. berghei, and characterize the functions of PSOP25 by genetic knockout (KO).

\section{Methods}

\section{Mice, parasites and mosquitoes}

Female BALB/c mice (six- to eight-week-old; Beijing Animal Institute, Beijing, China) were used for all animal experiments. P. berghei (ANKA strain 2.34) and $\Delta$ psop 25 lines (psop 25 gene knockout line) were maintained in mice and used for challenge infection. Adult Anopheles stephensi mosquitoes of the Hor strain were fed with $10 \%(\mathrm{w} / \mathrm{v})$ glucose solution and maintained in an insectary with a surrounding of $50-80 \%$ relative humidity, at $25^{\circ} \mathrm{C}$.

\section{Expression and purification of rPSOP25}

For the expression of full-length PSOP25, a psop25 fragment encoding aa 25-350 (excluding the signal peptide) was amplified from $P$. berghei genomic DNA with psop 25-F and psop25-R primers (Additional file 1: Table S1). The psop 25 fragment and the prokaryotic expression vector pET30a $(+)$ (Novagen, Darmstadt, Germany) were digested with restriction enzymes NdeI and HindIII, then ligation was performed using the Ligation High Kit (Toyobo, Osaka, Japan). The recombinant plasmid was transformed in Escherichia coli BL-21 (Novagen, Darmstadt, Germany) and the His-tagged 
recombinant PSOP25 (rPSOP25) was expressed at $20{ }^{\circ} \mathrm{C}$ for $12 \mathrm{~h}$ after induction with $1 \mathrm{mM}$ isopropyl- $\beta$-D-thiogalactopyranoside (Sigma-Aldrich, St. Louis, USA). For protein purification, cultures were harvested and lysed using binding buffer containing $10 \mathrm{mM}$ imidazole, $300 \mathrm{mM} \mathrm{NaCl}$ and $50 \mathrm{mM}$ sodium phosphate (pH 8.0) and treated by sonication (15 cycles of $20 \mathrm{~s}$ pulses and $30 \mathrm{~s}$ intervals). The soluble rPSOP25 was purified by Ni-NTA His-Bind Superflow (Novagen, Darmstadt, Germany), according to the manufacturer's instructions. Purified rPSOP25 was extensively desalted in $0.1 \mathrm{M}$ phosphate buffered saline (PBS, pH 7.4) overnight at $4{ }^{\circ} \mathrm{C}$, and then analyzed by SDS-PAGE.

\section{Animal immunization and monoclonal antibody (mAb) production}

To obtain polyclonal antisera against rPSOP25, a group of six female BALB/c mice were subcutaneously immunized with the purified protein $(50 \mu \mathrm{g} /$ mouse) emulsified in complete Freund's adjuvant (Sigma-Aldrich, St. Louis, USA), which has been used to produce high-titer antibodies [27]. Subsequently, the mice were given two booster immunizations of $25 \mu \mathrm{g}$ of rPSOP25 at 3-week intervals with the rPSOP25 protein emulsified in incomplete Freund's adjuvant (Sigma-Aldrich, St. Louis, USA). A group of negative control mice $(n=6)$ were immunized with PBS and same adjuvant formulations. For the final bleed, mouse blood was collected at 10 days after the final immunization by cardiac puncture and the antisera were obtained after the blood had clotted at room temperature. Antisera from individual mice were mixed together and used in the subsequent trials.

For monoclonal antibody (mAb) production, rPSOP25immunized $\mathrm{BALB} / \mathrm{c}$ mice were obtained as described above, then the spleen cells of the immunized mice were extracted and fused with Sp2/0-Ag14 myeloma cells to produce the anti-PSOP25 mAb [28]. The fused hybridoma cells were generated using the traditional polyethylene glycol method, and then selected in the hypoxanthineaminopterin-thymidine medium. The antibodies were screened by indirect antibody capture enzyme-linked immunosorbent assay (ELISA). The IgG fractions were prepared by ammonium sulfate precipitation, and then purified on a Protein A column (ThermoFisher Scientific, Waltham, USA), according to the manufacturer's instructions. The mAb isotype was determined by using the SBA Clonotyping $^{\text {ma }} \quad$ System-HRP (Southern Biotechnology Associates, Birmingham, USA).

\section{ELISA}

Antibody titers to rPSOP25 were determined by ELISA on day 14, 35 and 52 after the first immunization as previously described [26]. Briefly, 96-well plates were coated overnight with purified rPSOP25 at $4{ }^{\circ} \mathrm{C}$, and blocked with blocking buffer (0.05\% Tween 20 in $0.1 \mathrm{M}$ PBS, $1 \%$ bovine serum albumin, $\mathrm{pH} 7.4$ ) for $2 \mathrm{~h}$ at $37{ }^{\circ} \mathrm{C}$. The plates were then washed twice with PBS-T $(0.05 \%$ Tween 20 in $0.1 \mathrm{M} \mathrm{PBS}, \mathrm{pH} 7.4)$ and incubated with pooled mouse anti-rPSOP25 sera (1:200 dilution) in blocking buffer at $37{ }^{\circ} \mathrm{C}$ for $2 \mathrm{~h}$. After two washes, the wells were incubated for $2 \mathrm{~h}$ at $37^{\circ} \mathrm{C}$ with a 1:5000 dilution of HRP-conjugated goat anti-mouse IgG antibody (Invitrogen, Waltham, USA). After five final washes, tetramethyl benzidine (Amresco, Solon, USA) was added and the reaction was stopped by $2 \mathrm{mM} \mathrm{H}_{2} \mathrm{SO}_{4}$. The absorbance at $490 \mathrm{~nm}$ was measured with an ELISA plate reader.

For estimating the end point titer of immunized mice, sera from all mice in each immunization and control group were pooled and diluted from 1:200 to 1:204800 in a blocking buffer and incubated at $37{ }^{\circ} \mathrm{C}$ for $2 \mathrm{~h}$. The end point titers of the total IgG corresponded to the highest dilution at which the OD490 value was higher than the cut-off value, which was defined as the mean of the pooled negative control antisera $+3 \times$ standard deviation [29].

\section{Ookinete enrichment and lysate preparation}

The enrichment of ookinetes was referred to a modified protocol [30]. Briefly, $1.2 \mathrm{mg}$ phenylhydrazine (SigmaAldrich, St. Louis, USA) in $0.9 \% \mathrm{NaCl}$ were intraperitoneally (i.p.) injected into BALB/c mice 3 days before $P$. berghei infection. These treated mice were then i.p. injected with $5 \times 10^{6} \mathrm{P}$. berghei-infected red blood cells (iRBCs) to initiate the blood-stage infection. Parasitemia was allowed to reach approximately $1-3 \%$ at three days post-infection (p.i.), when the mice were anesthetized. After removal of the white blood cells, the infected blood was diluted 1:10 with the ookinete culture medium (100 mg/l neomycin, $50 \mathrm{mg} / \mathrm{l}$ streptomycin, $50 \mathrm{mg} / \mathrm{l}$ penicillin, $20 \%(\mathrm{v} / \mathrm{v}) \mathrm{FBS}$, and $1 \mathrm{mg} / \mathrm{l}$ heparin in RPMI 1640, pH 8.3) in a petri dish and maintained at $19{ }^{\circ} \mathrm{C}$ for $24 \mathrm{~h}$. The culture was then diluted in $45 \mathrm{ml}$ of $0.17 \mathrm{M} \mathrm{NH}_{4} \mathrm{Cl}$ on ice for $10 \mathrm{~min}$ to lyse erythrocytes. After a wash with 0.1 M PBS, ookinetes were separated on a $62 \%(\mathrm{v} / \mathrm{v})$ Nycodenz/PBS cushion by centrifugation $(1300 \times g)$ for $25 \mathrm{~min}$ at $25{ }^{\circ} \mathrm{C}$, treated with $0.15 \%$ saponin and washed once with $0.1 \mathrm{M}$ PBS. The ookinete lysate was prepared by resuspending the ookinetes in $2 \%$ SDS containing $1 \%$ Triton X-100 and $1 \times$ protease inhibitor cocktail (Roche, Castle Hill, Australia) for $30 \mathrm{~min}$ at room temperature.

\section{Western blot}

The parasite antigens $(10 \mu \mathrm{g})$ or purified rPSOP25 (500 ng) were subjected to electrophoresis under reducing or non-reducing conditions using a 10\% SDS-PAGE gel and electro-transferred to PVDF membrane (Bio- 
Rad, Hercules, USA). Western blot was performed essentially as described [26]. Primary antibodies were the pooled mouse anti-rPSOP25 serum (1:200) or antirPSOP25 mAb (1:1000), and HRP-conjugated goat antimouse IgG antibodies $(1: 10,000)$ (Invitrogen, Waltham, USA) were used as the secondary antibodies. Pbs 21 $\mathrm{mAb}$ (clone 13.1, 1:1000) was included as a positive control [31]. The sera (1:200) obtained from mice immunized with the PBS-adjuvant formulations were used as the negative control. The blot was developed using an ECL Western Blotting Kit (ThermoFisher Scientific, Waltham, USA).

\section{Indirect immunofluorescence assay (IFA)}

Parasites containing asexual stages, gametocytes, zygotes and ookinetes of $P$. berghei were fixed on slides [32]. Anti-rPSOP25 mAb (1:500) or Pbs21 mAb (clone 13.1, 1:500, positive control) or negative control sera (1:500) was incubated in 5\% skim milk and labeled with FITCconjugated goat anti-mouse IgG (1:500; Invitrogen, Waltham, USA) at $37^{\circ} \mathrm{C}$ for $1 \mathrm{~h}$. After staining of nuclei with 4', 6-diamidino-2-phenylindole (DAPI; Invitrogen, Waltham, USA), the slides were examined under Olympus BX53 (Olympus Corporation, Center Valley, USA) and the images were processed using Adobe Photoshop (Adobe Systems Inc., San Jose, USA).

\section{Quantification of TB activities}

For the in vitro assay, phenylhydrazine pre-treated mice were infected as described above. On day 3 p.i., parasitemia was determined, and $10 \mu \mathrm{l}$ of blood were taken from appropriate hosts and added to $90 \mu \mathrm{l}$ ookinete culture medium containing anti-rPSOP25 serum or negative control mouse serum at final dilutions of 1:5, 1:10, and 1:50. Additionally, anti-rPSOP25 $\mathrm{mAb}$ was added to the ookinete culture at 10,5 and $1 \mu \mathrm{g} / 100 \mu \mathrm{l}$ (concentration of $\mathrm{mAb}$ was $0.5 \mu \mathrm{g} / \mu \mathrm{l}$ ) of ookinete culture, respectively. Ookinete cultures were incubated at $19{ }^{\circ} \mathrm{C}$ for $24 \mathrm{~h}$ and the ookinete conversion rates were determined as described previously [26, 32].

For direct mosquito feeding assays (DFA), experimental mice $(n=3)$ were immunized with rPSOP25 and negative control mice $(n=3)$ were immunized with the PBS-adjuvant formulations as described above. Ten days after the final immunization, mice were infected i.p. with $5 \times 10^{6}$ P. berghei ANKA iRBC. For the antibody transfer experiment, three normal mice were injected intravenously with $150 \mu \mathrm{g}$ of anti-rPSOP $25 \mathrm{mAb} /$ mouse $1 \mathrm{~h}$ before mosquito feeding. Four-day-old female A. stephensi mosquitoes (starved for $12 \mathrm{~h}$ ) were allowed to feed on rPSOP25 immunized mice or antibody-transferred mice for $30 \mathrm{~min}$. Engorged mosquitoes were maintained in an insectary at $21{ }^{\circ} \mathrm{C}$ and $70 \%$ relative humidity. Ten days after feeding, $\sim 30$ mosquitoes were dissected, and midguts were stained with $0.5 \%$ mercurochrome (SigmaAldrich, St. Louis, USA) to count the number of oocysts per midgut.

\section{Generation of psop25 KO parasites}

To knock out all the protein-coding sequence of the psop 25 gene, the target vector containing an $h d h f r$ selection cassette was used (kindly provided by plasmoGEM, vector design ID, PbGEM-042760; http://plasmogem. sanger.ac.uk/). Before $P$. berghei transfection, vector DNA was digested by NotI followed by ethanol precipitation. The linearized plasmid $(10 \mu \mathrm{g})$ was electroporated into purified schizonts using the Nucleofector device as described previously [32]. After transfection, the complete parasite suspension was injected intravenously via the tail vein into mice. Following a $24 \mathrm{~h}$ incubation period, infected mice were treated for 3-4 days with pyrimethamine (Sigma-Aldrich, St. Louis, USA) via drinking water $(70 \mu \mathrm{g} / \mathrm{ml})$. Infected blood was collected and confirmed by integration-specific PCR (Additional file 1: Table S1). Monoclonal parasite lines were then obtained by limiting dilution.

\section{Phenotypic analysis of the $\Delta p$ sop 25 line}

To study whether deletion of psop 25 affected parasite growth, five phenylhydrazine-treated mice were inoculated i.p. with either $5 \times 10^{6}$ wildtype (WT) or $\Delta p s o p 25$ iRBCs. For each genotype, blood smears were used to monitor daily parasitemia, gametocytemia (mature gametocytes per $100 \mathrm{RBCs}$ ), and the gametocyte sex ratio (female: male ratio) [33]. To quantify male gamete exflagellation, $10 \mu \mathrm{l}$ of $P$. berghei-infected blood collected from mouse tail vein on day 3 p.i. were added into $90 \mu \mathrm{l}$ of ookinete culture medium and incubated for $15 \mathrm{~min}$ at $25{ }^{\circ} \mathrm{C}$, and the exflagellation centers were counted as previously described [32]. At the same time, ookinetes were cultured in vitro as described above [34], and the ookinete conversion rates were determined by IFA using a Pbs21 mAb [35]. For oocyst quantifications, mice at 3 days p.i. with each parasite line were fed to starved $A$. stephensi mosquitoes for $30 \mathrm{~min}$ [36, 37]. Ten days after feeding, 30 fed mosquitoes from each genotype were dissected for counting of the number of oocysts per infected mosquito and to determine the prevalence and intensity of infection.

\section{Statistical analysis}

Parasitemia, gametocytemia, gametocyte sex ratio and ookinete conversion rates between groups were analyzed by the Student's $t$-test, using GraphPad Prism software. The prevalence of infection (proportion of infected mosquito) was analyzed by the Fisher's exact test, while the intensity (number of oocysts per midgut) was analyzed by the Mann-Whitney $U$-test [38], 
using SPSS version 17.0. $P$-values less than 0.05 were considered statistically significant.

\section{Results}

The full-length rPSOP25 is immunogenic

A PSOP25 fragment which corresponded to aa 25-350 excluding the signal peptide was expressed in E. coli. This fragment included 14 predicted B cell epitopes [39] (Additional file 2: Figure S1). The purified rPSOP25 protein had a molecular size of $\sim 36.5 \mathrm{kDa}$ from SDSPAGE analysis, which was consistent with the predicted size of PSOP25 protein (Fig. 1a). To determine the immunogenicity of purified rPSOP25, we performed ELISA using pooled serum generated from mice immunized with the recombinant protein. The results showed that immunization with rPSOP25 induced strong antibody responses as compared to the negative control; the rPSOP25-specific IgG titers increased continuously during the course of vaccination (Student's $t$-test: $t_{(10)}=35.13, P<0.0001$; Fig. $1 \mathrm{~b}$ ). The antisera collected 10 days after the final immunization with rPSOP25 reached a titer of 1:1024000 (Fig. 1c). Meanwhile, an anti-rPSOP25 mAb was produced from a selected hybridoma line, which was determined to be the IgG1 isotype (Additional file 2: Figure S2).

\section{Anti-rPSOP25 antisera and $\mathrm{mAb}$ recognize the ookinete proteins}

The specificity of the pooled anti-rPSOP25 antisera and $\mathrm{mAb}$ was determined by Western blot against the rPSOP25 or protein lysate from ookinetes. On Western blots, both the antisera and mAb detected the $36.5 \mathrm{kDa}$ rPSOP25 (Fig. 2a) and a band of approximately $40 \mathrm{kDa}$ in the lysate of purified ookinetes under reducing conditions (Fig. 2a) and non-reducing conditions (Additional file 2: Figure S3), which is close to the predicted size of PSOP25. In a previous study, IFA using antisera raised against a partial domain of the PSOP25 protein indicated that PSOP25 is expressed on the surface of ookinetes [26]. Consistently, IFA with the anti-rPSOP25 mAb using zygotes, retorts and ookinetes without membrane permeabilization revealed strong fluorescence of the parasite body, which agrees with the surface localization of PSOP25 (Fig. 2b).

\section{Antibodies against PSOP25 show obvious TB activities}

Anti-rPSOP25 antisera and mAb were used in ookinete conversion assay to study the TB activity of the antibodies against PSOP25. When incubated with pooled mouse anti-rPSOP25 antisera or mAb, ookinete conversion was inhibited in a dose-dependent manner. In
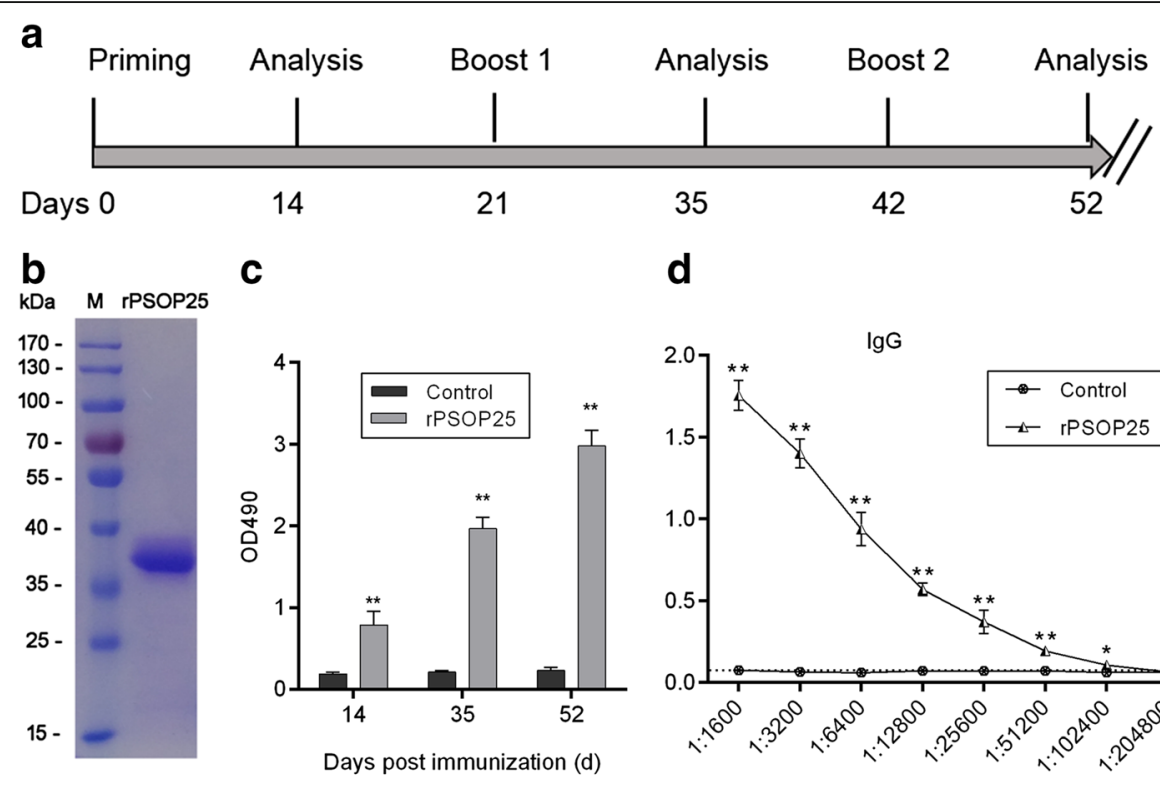

d

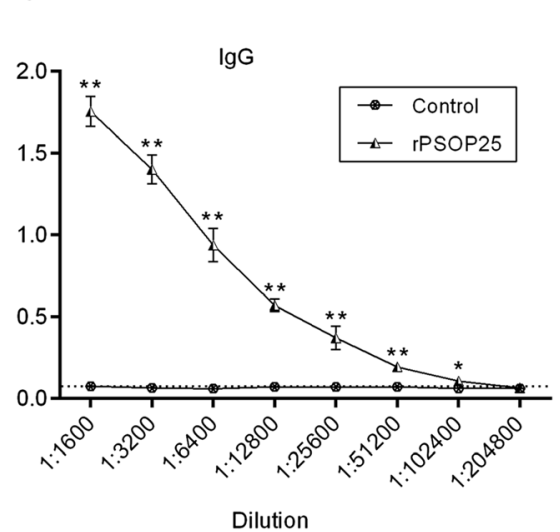

Fig. 1 a Mouse immunization and analysis scheme. b rPSOP25 was purified from E. coli and analyzed on a 10\% SDS-PAGE gel. c Antibody titers in immunized mice during immunization, experimental mice were immunized with rPSOP25 formulated in Freund's adjuvant; control mice were immunized with only Freund's adjuvant and 0.1 M PBS. The data represent two separate experiments. Error bar shows mean \pm standard deviation (SD). SD indicates the assay variance. ${ }^{* *} P<0.01$ (Student's $t$-test). $\mathbf{d}$ Anti-rPSOP25 total lgG titer at 10 days after the final immunization analyzed by ELISA. Mean of control antisera $+3 \times S D$ is shown by the broken lines. IgG titers correspond to the last dilution of the anti-rPSOP25 sera where in OD490 values were above the cut-off values. Cut-off value was defined as that of the pooled sera from control mice. The experiment was performed three times. Error bar shows mean $\pm \mathrm{SD} .{ }^{*} P<0.05$, ${ }^{* *} P<0.01$ (Student's $t$-test). Abbreviations: M, molecular weight marker; rPSOP25, purified rPSOP25 under reducing conditions 

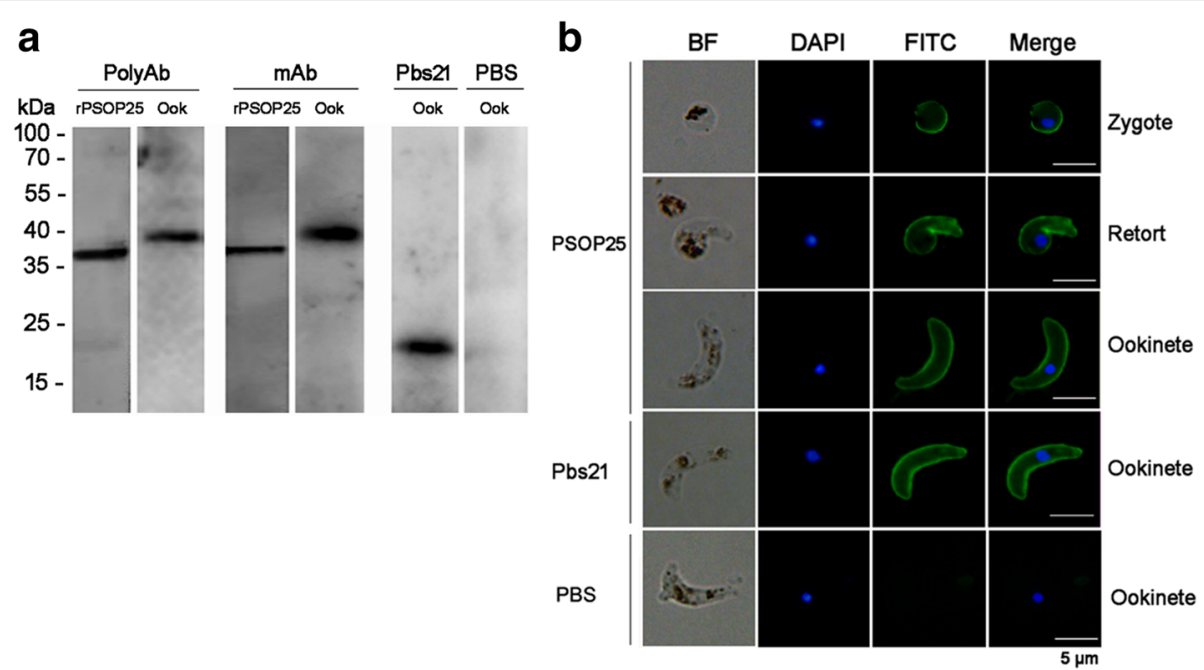

Fig. 2 a Western blot analysis of purified rPSOP25 protein and P. berghei ookinete (Ook) lysates with anti-rPSOP25 sera (PolyAb) and anti-rPSOP25 mAb. Lysates were subjected to electrophoresis under reducing conditions by SDS-PAGE. Pbs21 mAb was used as positive control; a control mouse serum (PBS) was used as negative control. b IFA was performed on zygote, retort and ookinete at different time points of ookinete culture using anti-rPSOP25 mAb (green - FITC). Positive control - Pbs21 mAb, negative control - a control mouse serum (PBS). Nuclei were labelled with DAPI (blue). BF, bright field. Scale-bars: $5 \mu \mathrm{m}$

ookinete cultures supplemented with the pooled immune sera at 1:5, 1:10 and 1:50 dilutions, ookinete conversion rates were reduced by $62.5 \%$ (Student's $t$-test: $t_{(4)}=22.52$, $P<0.0001), \quad 47.9 \%$ (Student's $t$-test: $t_{(4)}=21.44, \quad P<$ 0.0001 ), and $22.5 \%$ (Student's $t$-test: $t_{(4)}=9.11, P=0.0008$ ), respectively (Fig. 3a). Compared with the control sera, ookinete conversion rates in cultures with $\mathrm{mAb}$ added at 10,5 and $1 \mu \mathrm{g} / 100 \mu \mathrm{l}$ were reduced by $71.6 \%$ (Student's $t$ test: $t_{(4)}=32.04, P<0.0001$ ), $60.8 \%$ (Student's $t$-test: $t_{(4)}=$ 27.84, $P<0.0001$ ) and $32.0 \%$ (Student's $t$-test: $t_{(4)}=18.60$, $P<0.0001$ ), respectively (Fig. 3a).

To further examine the TB effect of anti-rPSOP25 antibodies in vivo, mice were immunized with rPSOP25 or passively transferred with the $\mathrm{mAb}$ and used in DFA.
Ten days after feeding, mosquitoes were dissected and midgut oocysts were counted. Mosquitoes fed on the rPSOP25-immunized mice showed a $31.2 \%$ reduction in the prevalence of oocysts, as compared to the control groups. The mean prevalence was $68.7 \%$ in mosquitoes fed on the rPSOP25-immunized mice, whereas it was 99.9\% in mosquitoes fed on the control mice (Fisher's exact test: $\mathrm{OR}=40.72,95 \% \mathrm{CI}=5.38-307.91, P<0.001$; Fig. 3b, Table 1). Moreover, mosquitoes fed on rPSOP25-immunized mice revealed a $66.3 \%$ reduction in oocyst density compared to the control group (MannWhitney $U$-test: $Z=-8.32, P<0.0001$; Fig. 3b, Table 1). Similarly, mosquitoes fed on the mice passively transferred with the mAb against PSOP25 had a $26.1 \%$
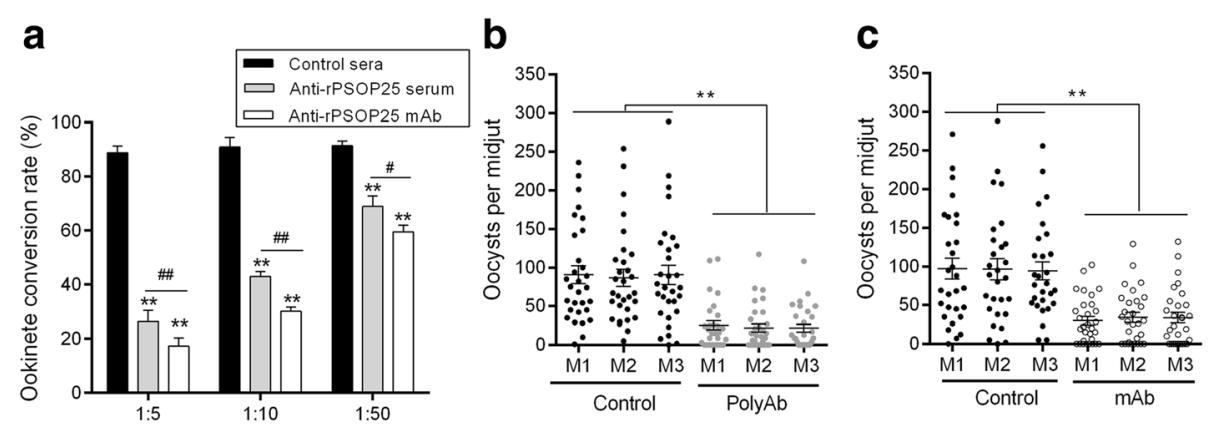

Fig. 3 a TB activities of anti-rPSOP25 serum and anti-rPSOP25 mAb on P. berghei ookinete formation in vitro. Anti-rPSOP25 serum, anti-rPSOP25 mAb, or normal mouse serum (control) were diluted at 1:5, 1:10 and 1:50, respectively. Means were representative of three independent experiments. Error bar shows mean \pm SD. ${ }^{* *}$ indicate significant difference compared with the control sera $(P<0.01)$. \# indicate significant difference between anti-rPSOP25 serum and mAb group $(P<0.05)$, \#\# $P<0.01$ (Student's $t$-test). b Direct mosquito feeding assay to assess the TB activity of polyclonal antisera in rPSOP25-immunized mice (3 mice per group). c Passive antibody transfer experiment to assess the TB activity of the anti-PSOP25 mAb. For $b$ and $c$, mosquito midguts were dissected at ten days post-infection, the number of oocysts was counted under a microscopy. The data are collated from three experiments. The mean number of oocysts and the SEM in each group are shown. ${ }^{*} P<0.01$ (Mann-Whitney U-test) 
Table 1 Evaluation of TB effect of anti-rPSOP25 serum and mAb in mosquito feeding assays

\begin{tabular}{|c|c|c|c|c|c|c|c|c|c|c|c|c|}
\hline & \multicolumn{6}{|c|}{ Immunization groups $^{\mathrm{a}}$} & \multicolumn{6}{|c|}{ Monoclonal antibody transfer groups ${ }^{\mathrm{b}}$} \\
\hline & \multicolumn{3}{|c|}{ Control mice } & \multicolumn{3}{|c|}{ rPSOP25 immunized mice } & \multicolumn{3}{|c|}{ Control mice } & \multicolumn{3}{|c|}{ mAb transferred mice } \\
\hline & M1 & M2 & M3 & M1 & M2 & M3 & M1 & M2 & M3 & M1 & M2 & M3 \\
\hline No. of mosquitoes infected/dissected & $30 / 30$ & $30 / 30$ & $29 / 30$ & $20 / 28$ & $20 / 30$ & $19 / 28$ & $28 / 29$ & $27 / 28$ & $28 / 28$ & $22 / 30$ & $21 / 29$ & $20 / 29$ \\
\hline Prevalence of infection $(\%)^{c}$ & 100 & 100 & 99.7 & 71.4 & 66.7 & 67.9 & 96.6 & 96.4 & 100 & 73.3 & 72.4 & 69.0 \\
\hline Mean prevalence (\%) & 99.9 & & & $68.7^{*}$ & & & & & 97.7 & & & $71.6^{*}$ \\
\hline Reduction in prevalence (\%) ${ }^{d}$ & & & & & & 31.2 & & & & & & 26.1 \\
\hline Oocyst intensity ${ }^{\mathrm{e}}$ & 90.7 & 86.8 & 90.5 & 25.2 & 22.1 & 21.7 & 97.1 & 96.3 & 94.3 & 29.8 & 34.2 & 33.7 \\
\hline SEM $^{f}$ & 11.58 & 11.17 & 12.50 & 5.890 & 5.232 & 5.138 & 13.47 & 13.66 & 11.82 & 5.462 & 6.373 & 6.800 \\
\hline Mean oocyst intensity & & & 89.3 & & & $23.0^{*}$ & & & 95.9 & & & $32.6^{*}$ \\
\hline Reduction in oocyst intensity $(\%)^{9}$ & & & & & & 66.3 & & & & & & 63.3 \\
\hline
\end{tabular}

${ }^{\mathrm{a}}$ TB activity assay was carried out using rPSOP25-immunized mice

${ }^{\mathrm{b}} \mathrm{TB}$ activity assay was carried out using BALB/C mice transferred with the anti-PSOP25 mAb

'The prevalence of infection was calculated by the number of mosquitoes with oocysts/total mosquitoes dissected in each group $\times 100 \%$

${ }^{\mathrm{d}}$ The percent reduction of prevalence was calculated as $\%$ mean prevalence control $-\%$ mean prevalence PSOP 25

${ }^{\mathrm{e}}$ Mean number of oocysts per mosquito midgut

f Standard error of the mean

${ }^{9}$ The percent reduction in oocyst intensity was calculated as (mean oocyst intensity control - mean oocyst intensity psop 25 )/mean oocyst intensity control $\times 100 \%$

${ }^{*} P<0.001$ for comparisons between the experimental group and the control group

reduction in the prevalence of oocysts (Fisher's exact test: $\mathrm{OR}=16.46,95 \% \mathrm{CI}=3.76-72.13, P<0.001$; Fig. $3 \mathrm{~b}$, Table 1) and a $63.3 \%$ reduction in density of oocysts (Mann-Whitney $U$-test: $Z=-6.97, P<0.0001$; Fig. 3b, Table 1).

\section{PSOP25 is required for the maturation of ookinetes}

To determine the function of PSOP25 during Plasmodium development, a psop 25 gene KO line ( $\Delta$ psop 25$)$ was generated in P. berghei (Fig. 4a) [40]. Genotypes of the cloned pyrimethamine-resistant parasites were confirmed by integration-specific PCR (Fig. 4b). To determine if psop 25 gene knockout led to any deficiencies in parasite development, we compared parasitemia, gametocytemia and gametocyte sex ratio between groups of BALB/c mice infected with $5 \times 10^{6} \Delta$ psop 25 or WT $P$. berghei parasites. Consistent with no expression of the PSOP25 protein in asexual erythrocytic stages, $\Delta$ psop 25 had no evident effect on asexual parasitemia (Fig. 5a). In addition, on day 3 p.i., gametocytemia and gametocyte sex ratio did not differ significantly between the WT parasite and the $\Delta$ psop 25 line (Fig. 5b, c). However, mean male gamete exflagellation events were slightly but significantly reduced in the $\Delta p s o p 25$ line, as compared to the WT parasites (Student's $t$-test: $t_{(10)}=4.01, P=$ 0.0024; Fig. 5d). Furthermore, in vitro ookinete cultures established from parasites at day 3 p.i. showed that ookinete conversion rate was significantly reduced in the $\Delta$ psop 25 line, although the mature ookinetes in the $\Delta$ psop25 line appeared morphologically normal (data not shown). Using reverse-transcriptase-PCR, we determined that there was no psop 25 expression in the ookinetes of
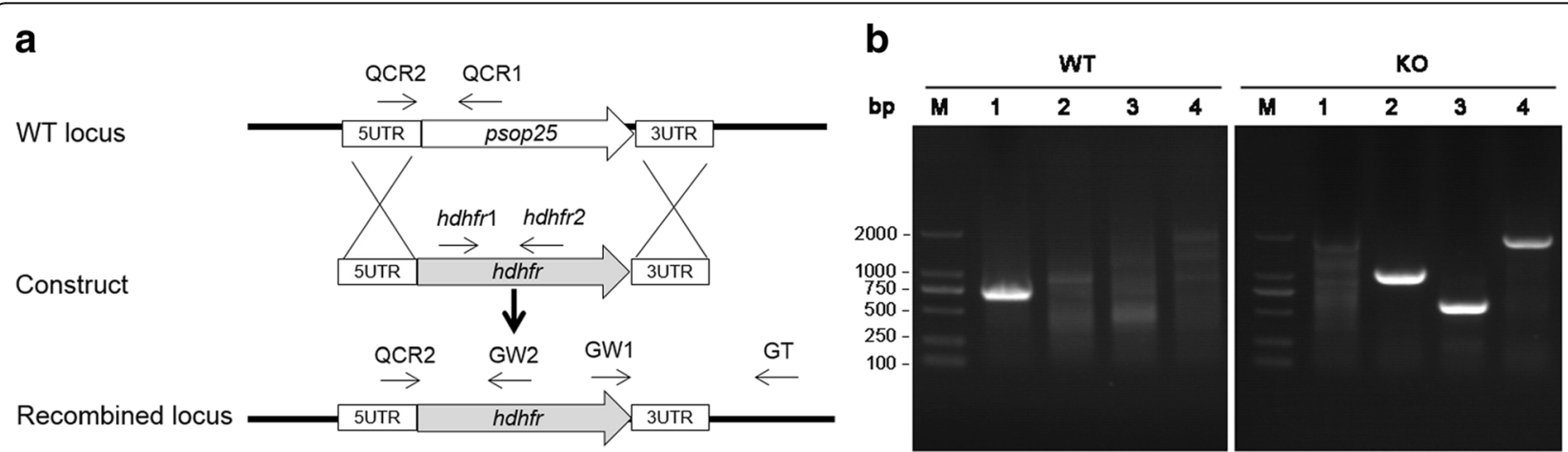

Fig. 4 a Schematic representation of the WT locus, the construct used for transfection and the recombined locus with psop25 replaced with the hdhfr cassette. Primers QCR1, QCR2, GW2, hdhfr1, hdhfr2, GW1 and GT used for diagnostic PCR of the WT locus or knockout are marked. $\mathbf{b}$ Lane 1: primers QCR1 + QCR2 (696 bp) are used for diagnostic PCR of the WT locus. Lanes 2, 3 and 4 are PCR products from primers GW2 + QCR2 (1,002 bp), hdhfr1 + hdhfr2 (561 bp), GW1 + GT (1,831 bp) for PCR verification of psop25 KO, respectively 

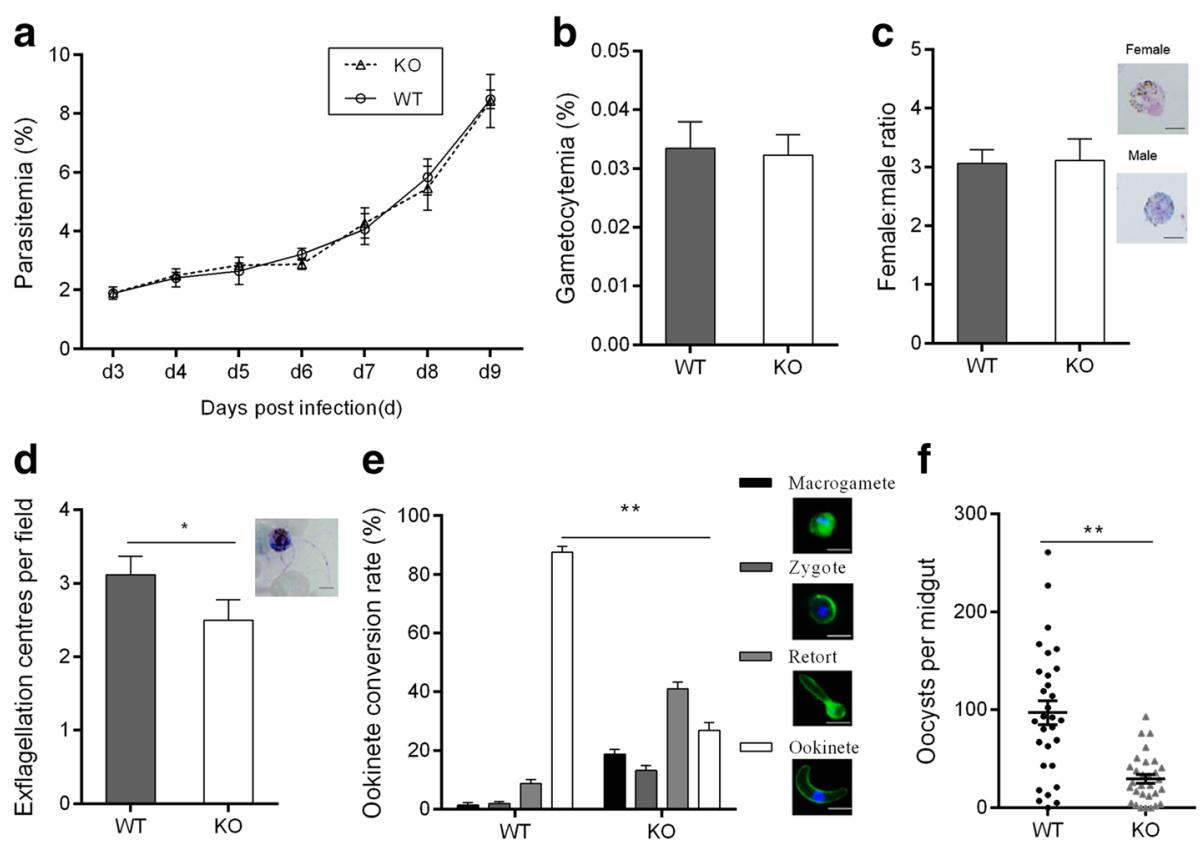

Fig. 5 a Average parasitemia was calculated in mice after infection with the wild-type (WT) or $\Delta p$ sop25 parasites $(n=5)$. $\mathbf{b}$ Gametocytemia in mice infected with WT or $\Delta$ psop25 parasites $(n=3)$. c Female: male gametocyte ratios of WT or $\Delta p$ sop25 parasites $(n=3)$. $\mathbf{d}$ Exflagellation of WT and $\Delta p s o p 25$ microgametes $(n=3),{ }^{*} P<0.05$ (Student's t-test). e Ookinete conversion rates in vitro of WT and $\Delta p s o p 25$ parasites. For $\mathbf{c}$, $\mathbf{d}$ and $\mathbf{e}$, characteristic morphologies of parasites are shown on the right $(n=3),{ }^{* *} P<0.01$ (Student's $t$-test). $\mathbf{f}$ Oocyst number per midgut in mosquitoes 10 days after feeding on mice infected with the WT and the $\Delta$ psop25 parasites. The horizontal bar shows the mean number of oocysts per midgut in mosquito $\left( \pm\right.$ SEM). ${ }^{* *} P<0.01$ (Mann-Whitney U-test). For a-f, all the data are representative of three separate experiments. Scale-bars: $5 \mu \mathrm{m}$

the $\Delta$ psop 25 line, further confirming that psop 25 was deleted (data not shown). In the WT line, the zygote, retort, and ookinete conversion rates was 2.0, 8.8 and $87.74 \%$, respectively. Whereas in the $\Delta$ psop 25 line, 13.3 and $41.0 \%$ parasites progressed to the zygote and retort stages, respectively, further maturation to ookinetes was reduced by $60.9 \%$ (Student's $t$-test: $t_{(4)}=$ 31.69, $P<0.0001$; Fig. 5e), indicating that PSOP25 might play a crucial role in ookinete maturation. The oocyst density was reduced to 29.7 per mosquito midgut in those fed on the $\Delta$ psop 25 parasites as compared to 96.9 in WT parasites, reflecting a $69.4 \%$ reduction (Mann-Whitney $U$-test: $Z=-4.25, P<0.0001$; Fig. 5f, Table 2).

\section{Discussion}

Disrupting the parasite life-cycle to prevent the disease from being transmitted to other individuals represents a key component of an integrated malaria control strategy [41]. Despite investigations on several TBV antigens over the last 40 years, there is still a need to discover new TBV vaccine candidates for malaria elimination purpose [42]. In our previous study, we evaluated the transmissionblocking activities of a partial 200 aa PSOP25 domain [26]. Here we expressed the full-length rPSOP25 protein and raised polyclonal antisera as well as $\mathrm{mAb}$ for this protein, which were found to possess effective TB activities in an in vitro ookinete formation assay and in vivo DFA.

Antibody concentrations against TBV candidates, as measured by conventional ELISA, have been shown to be associated with the effectiveness of TB activities in mosquito membrane feeding assays $[19,43]$. In our previous study, the 200 aa PSOP25 fragment including ten predicted antibody epitopes had elicited obvious antibody responses, and immunized mouse antisera produced TB activities with $25 \%$ reduction in prevalence

Table 2 Oocyst number per midgut in mosquitoes 10 days after feeding on mice infected with the WT and the $\Delta p s o p 25$ parasites

\begin{tabular}{llllcc}
\hline & $\begin{array}{l}\text { No. of mosquitoes } \\
\text { (infected/dissected) }\end{array}$ & $\begin{array}{l}\text { Prevalence of } \\
\text { infection (\%) }\end{array}$ & Mean oocyst (IQR) & SEM $^{\mathrm{c}}$ & $\begin{array}{l}\text { Reduction in oocyst } \\
\text { intensity (\%) }\end{array}$ \\
\hline Wild type & $29 / 30$ & 99.7 & $96.93(43.00-139.8)$ & 12.01 & \\
Spsop25 & $27 / 30$ & 90 & $29.67(10.25-43.25)$ & 4.481 & 67.26 \\
\hline
\end{tabular}

${ }^{a}$ The prevalence of infection was calculated by the number of mosquitoes with oocysts/total mosquitoes dissected in each group $\times 100 \%$

${ }^{\mathrm{b}} \mathrm{IQR}$, inter-quartile range

'Standard error of the mean

${ }^{\mathrm{d}}$ The percent reduction in oocyst intensity was calculated as (mean oocyst intensity $\mathrm{wT}^{-}$mean oocyst intensity ${ }_{\Delta p s o p 25}$ )/mean oocyst intensity $\mathrm{wT}_{\mathrm{m}} \times 100 \%$ 
and $64.3 \%$ reduction in oocyst density [26]. The fulllength PSOP25 is predicted to contain four additional antibody epitopes, and the full-length rPSOP25 indeed induced high antibody titers in mice. In parallel comparison experiments, mosquitoes fed on mice immunized with the full-length and partial rPSOP25 showed similar levels $(\sim 60 \%)$ of reduction in oocyst density as compared to those fed on control mice. However, there was a greater degree of reduction in oocyst prevalence in mosquitoes fed on mice immunized with the full-length protein $(31.2 \%)$ as compared to that in mosquitoes fed on mice immunized with the partial rPSOP25 (25\%) [26]. The TB activities of PSOP25 were comparable to those of PSOP12 [44], PSOP7 and PSOP26 [26] in the reduction of oocyst density and infection prevalence in DFA. Furthermore, monoclonal antibodies have been a valuable tool for the characterization of TBVs $[45,46]$. Previous studies have explored passive transfer of transmission blocking mAbs (e.g. Pbs21 mAb clone 13.1) for $\mathrm{TB}$ activities [31, 47]. In this study, an IgGl-type mAb against PSOP25 significantly inhibited the development of ookinetes and oocyst when administered through passive transfer prior to mosquito feeding, and the TB activities were comparable to those from the full-length rPSOP25 immunization group. Passive immunization with $\mathrm{TB}$ mAbs may be of additional values as an intervention in specific circumstances, including malaria epidemic settings [48, 49].

Transmission-blocking strategies require improved knowledge of the basic biology of the parasite [50]. Recent efforts in genomics, transcriptomics and proteomics have revealed a large number of molecules that may play key roles in ookinete development [51-53]. Further, screening for novel vaccine candidates based on gene $\mathrm{KO}$ and phenotypic analysis will undoubtedly yield valuable information regarding the cell biology of the ookinetes [52]. Several ookinete proteins which play various roles in midgut colonization have been characterized, including the GPI-anchored P25 and P28 proteins [20, 54], circumsporozoite TRAP-related protein (CTRP) $[55,56]$, Plasmodium von Willebrand factor A domain-related protein (WARP) [57], secreted ookinete apical protein (SOAP) [55, 58], and the recently described putative secreted ookinete proteins (PSOPs) [52]. Here, we generated a $\triangle p s o p 25$ line, and detected slightly reduced exflagellation activity of male gametocytes, but significantly reduced ookinete conversion rate in vitro. Whereas most $\Delta$ psop 25 parasites progressed normally to zygote and retort stages, further maturation to ookinetes was retarded, which resulted in a $60.9 \%$ reduction in the number of ookinetes as compared to the WT parasite. This phenotype shows some similarity with that of psop2 knockout parasites, which appeared morphologically normal but showed reduced in vivo ookinete numbers
[52]. The blockade in ookinete maturation in the $\Delta$ psop 25 parasite was further reflected in the reduction of oocyst density in DFA. The oocysts per mosquito midgut in those fed on the $\Delta$ psop 25 parasites was reduced by $69.4 \%$, like that with the $\Delta p s o p 9$ line [52]. Given that other PSOP proteins such as PSOP26 showed ookinete surface localization [26] and there is a possibility that these surface proteins interact, it would be interesting to determine whether psop 25 disruption could affect the expression of other PSOP proteins.

\section{Conclusions}

This study confirmed that the full-length recombinant protein of a newly identified TBV candidate PSOP25 expressed in ookinetes of the rodent parasite $P$. berghei could also elicit a strong antibody response in mice. Both polyclonal mouse antisera and $\mathrm{mAb}$ against this protein recognized the surface of zygotes, retorts and ookinetes and possessed similar TB activities as the polyclonal antisera generated against the truncated version of this protein. Genetic KO study indicated that PSOP25 in $P$. berghei is required for ookinete formation and maturation. Collectively, PSOP25 is an excellent TBV candidate targeting the post-fertilization stages, and further assessment of TB activities in P. falciparum and P. vivax are warranted.

\section{Additional files}

Additional file 1: Table S1. Primer information and sequences. (DOCX $15 \mathrm{~kb}$ )

Additional file 2: Figure S1. Predicted B cell epitopes of the PSOP25 protein (http://tools.iedb.org/bcell). Below is the protein domain architecture of PSOP25 with signal peptide highlighted in red, low complexity in pink, and transmembrane region in blue. Figure S2. The isotype of anti-rPSOP25 mAb was identified by ELISA using by the SBA Clonotyping ${ }^{\text {TM }}$ System-HRP. The data represent two separate experiments. Error bar shows mean + standard deviation. Figure S3. Western blot analysis of $P$. berghei ookinete lysates with anti-rPSOP25 sera (PolyAb) and anti-rPSOP25 $\mathrm{mAb}$. Lysates were subjected to electrophoresis under non-reducing conditions by SDS-PAGE. Pbs21 mAb was used as positive control; a control mouse serum (PBS) was used as negative control. (ZIP $2413 \mathrm{~kb}$ )

\begin{abstract}
Abbreviations
CTRP: Circumsporozoite TRAP-related protein; ELISA: Enzyme-linked immunosorbent assay; GPI: Glycosyl-phosphatidylinositol; i.p.: Intraperitoneally; IFA: Indirect immunofluorescence assay; iRBCs: Infected red blood cells; KO: Knockout; mAb: Monoclonal antibody; PBS: Phosphate-buffered saline; PBS-T: 0.05\% Tween 20 in phosphate-buffered saline; PSOPs: Putative secreted ookinete proteins; RBCs: Red blood cells; SOAP: Secreted ookinete apical protein; TBS-T: 0.1\% Tween 20 in Tris-buffered saline; TBV: Transmission-blocking vaccine; WARP: von Willebrand factor A domain-related protein; WT: Wildtype
\end{abstract}

\section{Acknowledgements}

We are grateful to Ms. Jun Liu for technical support and to Dr. Hiroyuki Matsuoka for providing the Pbs21 mAb clone 13.1. We thank plasmoGEM for kindly providing the target vector PbGEM-042760 (http://plasmogem.sanger.ac.uk). 


\section{Funding}

This study was supported by the National Institutes of Health grants R01Al099611 and R01Al104946, and the National Natural Science Foundation of China 81471978.

\section{Availability of data and materials}

The data supporting the conclusions of this article are included within the article

\section{Authors' contributions}

YC and LC conceived the study and helped draft the manuscript. EL, GBH and TT helped with the bioinformatics analysis and drafted the manuscript. WZ carried out the rPSOP25 protein expression, mAb development, TB activity studies of PSOP25 and drafted the manuscript. FL and YH carried out function studies by genetic knockout, statistical analysis. QL and QF participated in the antibodies specificity detection and statistical analysis. All authors contributed to the writing of the manuscript, read and approved the final manuscript.

\section{Competing interests}

The authors declare that they have no competing interests.

\section{Consent for publication}

Not applicable.

\section{Ethics approval and consent to participate}

Animal use was carried out according to the guidelines of the animal ethics committee of China Medical University.

\section{Author details}

${ }^{1}$ Department of Pathogen Biology, College of Basic Medical Sciences, China Medical University, Shenyang, Liaoning 110001, China. ${ }^{2}$ Laboratory of Surgery, The Affiliated Hospital, Inner Mongolia Medical University, Hohhot 010050, China. ${ }^{3}$ Department of Immunology, College of Basic Medical Sciences, China Medical University, Shenyang, Liaoning 110001, China. ${ }^{4}$ Department of Entomology, The Pennsylvania State University, University Park, PA 16802, USA. ${ }^{5}$ Cell-free Science and Technology Research Center, Ehime University, Matsuyama, Ehime 790-8577, Japan. ${ }^{6}$ Dalian Institute of Biotechnology, Dalian, Liaoning, China.

\section{Received: 4 July 2016 Accepted: 6 December 2016}

Published online: 05 January 2017

\section{References}

1. WHO. World malaria report. 2015. http://www.who.int/malaria/publications/ world-malaria-report-2015/report/en/.

2. Miotto O, Almagro-Garcia J, Manske M, Macinnis B, Campino S, Rockett KA, et al. Multiple populations of artemisinin-resistant Plasmodium falciparum in Cambodia. Nat Genet. 2013;45(6):648-55.

3. Ariey F, Witkowski B, Amaratunga C, Beghain J, Langlois AC, Khim N, et al. A molecular marker of artemisinin-resistant Plasmodium falciparum malaria. Nature. 2014;505(7481):50-5.

4. Mueller I, Galinski MR, Baird JK, Carlton JM, Kochar DK, Alonso PL, del Portillo HA. Key gaps in the knowledge of Plasmodium vivax, a neglected human malaria parasite. Lancet Infect Dis. 2009;9(9):555-66.

5. Smith $T A$, Leuenberger $\mathrm{R}$, Lengeler $\mathrm{C}$. Child mortality and malaria transmission intensity in Africa. Trends Parasitol. 2001;17(3):145-9.

6. Kaslow DC. Transmission-blocking vaccines. Chem Immunol. 2002;80:287-307.

7. Eckhoff $P$. Mathematical models of within-host and transmission dynamics to determine effects of malaria interventions in a variety of transmission settings. Am J Trop Med Hyg. 2013;88(5):817-27.

8. Outchkourov NS, Roeffen W, Kaan A, Jansen J, Luty A, Schuiffel D, et al. Correctly folded Pfs48/45 protein of Plasmodium falciparum elicits malaria transmissionblocking immunity in mice. P Natl Acad Sci USA. 2008;105(11):4301-5.

9. Singh SK, Roeffen W, Andersen G, Bousema T, Christiansen M, Sauerwein R, et al. A Plasmodium falciparum 48/45 single epitope R0.6C subunit protein elicits high levels of transmission blocking antibodies. Vaccine. 2015;33(16):1981-6.

10. MacDonald NJ, Nguyen V, Shimp R, Reiter K, Herrera R, Burkhardt M, et al. Structural and Immunological Characterization of Recombinant 6-Cysteine Domains of the Plasmodium falciparum Sexual Stage Protein Pfs230. J Biol Chem. 2016;291(38):19913-22.
11. Tachibana M, Wu Y, Iriko H, Muratova O, MacDonald NJ, Sattabongkot J, et al. N-terminal prodomain of Pfs230 synthesized using a cell-free system is sufficient to induce complement-dependent malaria transmission-blocking activity. Clin Vaccine Immunol. 2011;18(8):1343-50.

12. Lee SM, Wu CK, Plieskatt J, McAdams DH, Miura K, Ockenhouse C, King CR. Assessment of Pfs 25 expressed from multiple soluble expression platforms for use as transmission-blocking vaccine candidates. Malar J. 2016;15(1):405.

13. Chaurio RA, Pacheco MA, Cornejo OE, Durrego E, Stanley Jr CE, Castillo Al, et al. Evolution of the Transmission-Blocking Vaccine Candidates Pvs28 and Pvs25 in Plasmodium vivax: Geographic Differentiation and Evidence of Positive Selection. PLoS Negl Trop Dis. 2016;10(6):e0004786.

14. Roeffen $W$, Theisen M, van de Vegte-Bolmer M, van Gemert G, Arens T, Andersen $\mathrm{G}$, et al. Transmission-blocking activity of antibodies to Plasmodium falciparum GLURP.10C chimeric protein formulated in different adjuvants. Malar J. 2015;14:443.

15. Miura K, Stone WJ, Koolen KM, Deng B, Zhou L, van Gemert GJ, et al. An inter-laboratory comparison of standard membrane-feeding assays for evaluation of malaria transmission-blocking vaccines. Malar J. 2016;15:463.

16. Jones S, Grignard L, Nebie I, Chilongola J, Dodoo D, Sauerwein R, et al. Naturally acquired antibody responses to recombinant Pfs230 and Pfs48/45 transmission blocking vaccine candidates. J Infect. 2015;71(1):117-27.

17. Sinden RE. A proteomic analysis of malaria biology: integration of old literature and new technologies. Int J Parasitol. 2004;34(13-14):1441-50.

18. Stone WJ, Dantzler KW, Nilsson SK, Drakeley CJ, Marti M, Bousema T, et al. Naturally acquired immunity to sexual stage $P$. falciparum parasites. Parasitology. 2016;143(2):187-98.

19. Vermeulen AN, Ponnudurai T, Beckers PJ, Verhave JP, Smits MA, Meuwissen $J$ H. Sequential expression of antigens on sexual stages of Plasmodium falciparum accessible to transmission-blocking antibodies in the mosquito. J Exp Med. 1985;162(5):1460-76.

20. Tomas AM, Margos G, Dimopoulos G, van Lin LH, de Koning-Ward TF, Sinha R, et al. P25 and P28 proteins of the malaria ookinete surface have multiple and partially redundant functions. Embo J. 2001;20(15):3975-83.

21. Tirawanchai N, Winger LA, Nicholas J, Sinden RE. Analysis of immunity induced by the affinity-purified 21-kilodalton zygote-ookinete surface antigen of Plasmodium berghei. Infect Immun. 1991;59(1):36-44.

22. Gregory JA, Li F, Tomosada LM, Cox CJ, Topol AB, Vinetz JM, et al. Algaeproduced Pfs 25 elicits antibodies that inhibit malaria transmission. PLoS One. 2012;7(5):e37179.

23. Kumar R, Angov E, Kumar N. Potent malaria transmission-blocking antibody responses elicited by Plasmodium falciparum Pfs 25 expressed in Escherichia coli after successful protein refolding. Infect Immun. 2014;82(4):1453-9.

24. Blagborough AM, Musiychuk K, Bi H, Jones RM, Chichester JA, Streatfield S, et al. Transmission blocking potency and immunogenicity of a plantproduced Pvs25-based subunit vaccine against Plasmodium vivax. Vaccine. 2016;34(28):3252-9.

25. Otto TD, Bohme U, Jackson AP, Hunt M, Franke-Fayard B, Hoeijmakers WA et al. A comprehensive evaluation of rodent malaria parasite genomes and gene expression. BMC Biol. 2014;12:86.

26. Zheng W, Kou X, Du Y, Liu F, Yu C, Tsuboi T, et al. Identification of three ookinete-specific genes and evaluation of their transmission-blocking potentials in Plasmodium berghei. Vaccine. 2016;34(23):2570-8.

27. Hunter RL, Lal AA. Copolymer adjuvants in malaria vaccine development. Am J Trop Med Hyg. 1994;50(4 Suppl):52-8.

28. Kohler G, Milstein C. Continuous cultures of fused cells secreting antibody of predefined specificity. Nature. 1975;256(5517):495-7.

29. Chowdhury DR, Angov E, Kariuki T, Kumar N. A potent malaria transmission blocking vaccine based on codon harmonized full length Pfs48/45 expressed in Escherichia coli. PLoS One. 2009;4(7):e6352.

30. Sinden RE, Hartley RH, Winger L. The development of Plasmodium ookinetes in vitro: an ultrastructural study including a description of meiotic division. Parasitology. 1985;91(Pt 2):227-44.

31. Winger LA, Tirawanchai N, Nicholas J, Carter HE, Smith JE, Sinden RE. Ookinete antigens of Plasmodium berghei. Appearance on the zygote surface of an Mr $21 \mathrm{kD}$ determinant identified by transmission-blocking monoclonal antibodies. Parasite Immunol. 1988;10(2):193-207.

32. Kou X, Zheng W, Du F, Liu F, Wang M, Fan Q, et al. Characterization of a Plasmodium berghei sexual stage antigen $\mathrm{PbPH}$ as a new candidate for malaria transmission-blocking vaccine. Parasit Vectors. 2016;9(1):190.

33. Guttery DS, Poulin B, Ramaprasad A, Wall RJ, Ferguson DJ, Brady D, et al. Genome-wide functional analysis of Plasmodium protein phosphatases 
reveals key regulators of parasite development and differentiation. Cell Host Microbe. 2014;16(1):128-40.

34. Janse CJ, Mons B, Rouwenhorst RJ, Van der Klooster PF, Overdulve JP, Van der Kaay $\mathrm{HJ}$. In vitro formation of ookinetes and functional maturity of Plasmodium berghei gametocytes. Parasitology. 1985;91(Pt 1):19-29.

35. Reininger L, Billker O, Tewari R, Mukhopadhyay A, Fennell C, DorinSemblat D, et al. A NIMA-related protein kinase is essential for completion of the sexual cycle of malaria parasites. J Biol Chem. 2005;280(36): 31957-64.

36. Doi M, Tanabe K, Tachibana S, Hamai M, Tachibana M, Mita T, et al. Worldwide sequence conservation of transmission-blocking vaccine candidate Pvs230 in Plasmodium vivax. Vaccine. 2011;29(26):4308-15.

37. Hills T, Srivastava A, Ayi K, Wernimont AK, Kain K, Waters AP, et al. Characterization of a new phosphatase from Plasmodium. Mol Biochem Parasit. 2011;179(2):69-79.

38. Blagborough AM, Yoshida S, Sattabongkot J, Tsuboi T, Sinden RE. Intranasal and intramuscular immunization with Baculovirus Dual Expression Systembased Pvs 25 vaccine substantially blocks Plasmodium vivax transmission. Vaccine. 2010;28(37):6014-20.

39. Kolaskar AS, Tongaonkar PC. A semi-empirical method for prediction of antigenic determinants on protein antigens. FEBS Lett. 1990;276(1-2): $172-4$.

40. Janse CJ, Franke-Fayard B, Mair GR, Ramesar J, Thiel C, Engelmann S, et al. High efficiency transfection of Plasmodium berghei facilitates novel selection procedures. Mol Biochem Parasitol. 2006;145(1):60-70.

41. Goodman AL, Blagborough AM, Biswas S, Wu Y, Hill AV, Sinden RE, et al. A viral vectored prime-boost immunization regime targeting the malaria Pfs 25 antigen induces transmission-blocking activity. PLoS One. 2011;6(12):e29428.

42. mal ERACGoV. A research agenda for malaria eradication: vaccines. PLOS Med. 2011;8(1):e1000398.

43. Nikolaeva D, Draper SJ, Biswas S. Toward the development of effective transmission-blocking vaccines for malaria. Expert Rev Vaccines. 2015; 14(5):653-80.

44. Sala KA, Nishiura H, Upton LM, Zakutansky SE, Delves MJ, lyori M, et al. The Plasmodium berghei sexual stage antigen PSOP12 induces anti-malarial transmission blocking immunity both in vivo and in vitro. Vaccine. 2015; 33(3):437-45.

45. Vermeulen AN, Roeffen WF, Henderik JB, Ponnudurai T, Beckers PJ, Meuwissen JH. Plasmodium falciparum transmission blocking monoclonal antibodies recognize monovalently expressed epitopes. Dev Biol Stand. 1985;62:91-7.

46. Carter R. Transmission blocking malaria vaccines. Vaccine. 2001;19(17-19): 2309-14.

47. Ranawaka GR, Fleck SL, Blanco AR, Sinden RE. Characterization of the modes of action of anti-Pbs21 malaria transmission-blocking immunity: ookinete to oocyst differentiation in vivo. Parasitology. 1994;109(Pt 4):403-11.

48. Sauerwein RW. Malaria transmission-blocking vaccines: the bonus of effective malaria control. Microbes Infect. 2007;9(6):792-5.

49. Sauerwein RW, Bousema T. Transmission blocking malaria vaccines: Assays and candidates in clinical development. Vaccine. 2015;33(52):7476-82.

50. Tewari R, Straschil U, Bateman A, Bohme U, Cherevach I, Gong P, et al. The systematic functional analysis of Plasmodium protein kinases identifies essential regulators of mosquito transmission. Cell Host Microbe. 2010;8(4):377-87.

51. Hall N, Karras M, Raine JD, Carlton JM, Kooij TW, Berriman M, et al. A comprehensive survey of the Plasmodium life cycle by genomic, transcriptomic, and proteomic analyses. Science. 2005;307(5706):82-6.

52. Ecker A, Bushell ES, Tewari R, Sinden RE. Reverse genetics screen identifies six proteins important for malaria development in the mosquito. Mol Microbiol. 2008;70(1):209-20.

53. Lal K, Prieto JH, Bromley E, Sanderson SJ, Yates 3rd JR, Wastling JM, et al. Characterisation of Plasmodium invasive organelles; an ookinete microneme proteome. Proteomics. 2009;9(5):1142-51.

54. Sinden RE, Billingsley PF. Plasmodium invasion of mosquito cells: hawk or dove? Trends Parasitol. 2001;17(5):209-12.

55. Nacer A, Underhill A, Hurd $H$. The microneme proteins CTRP and SOAP are not essential for Plasmodium berghei ookinete to oocyst transformation in vitro in a cell free system. Malar J. 2008;7:82.

56. Ramakrishnan C, Dessens JT, Armson R, Pinto SB, Talman AM, Blagborough $A M$, et al. Vital functions of the malarial ookinete protein, CTRP, reside in the A domains. Int J Parasitol. 2011:41(10):1029-39.
57. Yuda M, Yano K, Tsuboi T, Torii M, von Willebrand CY, Factor A. von Willebrand Factor A domain-related protein, a novel microneme protein of the malaria ookinete highly conserved throughout Plasmodium parasites. Mol Biochem Parasitol. 2001;116(1):65-72.

58. Dessens JT, Siden-Kiamos I, Mendoza J, Mahairaki V, Khater E, Vlachou D, et al. SOAP, a novel malaria ookinete protein involved in mosquito midgut invasion and oocyst development. Mol Microbiol. 2003;49(2):319-29.

\section{Submit your next manuscript to BioMed Central and we will help you at every step:}

- We accept pre-submission inquiries

- Our selector tool helps you to find the most relevant journal

- We provide round the clock customer support

- Convenient online submission

- Thorough peer review

- Inclusion in PubMed and all major indexing services

- Maximum visibility for your research

Submit your manuscript at www.biomedcentral.com/submit
Biomed Central 